\title{
EL PAPEL DE LA VITAMINA D EN LA RESPUESTA INMUNE Y EN LAS ENFERMEDADES ALÉRGICAS
}

\author{
THE ROLE OF VITAMIN D IN THE IMMUNE RESPONSE AND \\ ALLERGICDISEASES
}

\author{
Meza-Torres Catherine ${ }^{1}$ \\ Marrugo-Cano Javier ${ }^{2}$
}

Correspondencia: jmarrugoc@unicartagena.edu.co

Recibido para evaluación: noviembre - 1 - 2015. Aceptado para publicación: noviembre - 10 - 2015

\section{RESUMEN}

Introducción: la vitamina $\mathrm{D}$ es una de las moléculas más pleiotrópicas. Juega un papel importante en el metabolismo del calcio, en la salud pulmonar y en el sistema inmune. En estudios epidemiológicos se ha relacionado la insuficiencia de esta vitamina con el asma y la dermatitis atópica. Así mismo, algunos estudios genéticos que incluyen hasta el rastreo genómico han reportado asociación entre el receptor de la vitamina D (VDR) y el asma.

Objetivo: identificar el papel de la vitamina $D$ en la respuesta inmune $y$ en las enfermedades alérgicas.

Metodología: se realizó una búsqueda electrónica en las bases de datos PubMed, ScienceDirect, Protein data Bank, NCBI, Blackwell Synergy Wiley Online Library.

Resultados: se selecionaron 120 articulos para la revision y se escogieron 76 articulos completos y dos resúmenes de nuestro grupo.

Conclusión: estudios epidemiológicos y de genética han relacionado a la vitamina $D$ y su receptor (VDR) con el desarrollo de las enfermedades alérgicas. Esta evidencia es extensa y en ocasiones contradictoria, la cual se podría explicar por el reclutamiento diferencial de coactivadores del complejo VD-VDR-RXR. Sin embargo, la mayoría de los estudios experimentales in vitro e in vivo demuestran que la vitamina $D$ ejerce un efecto modulador sobre diversos tipos de células del sistema inmune innato y adaptativo, así como sobre las células involucradas en la respuesta inmune del tipo Th1, Th2, Treg y Th17. Se concluye que esta vitamina juega un papel fundamental sobre las respuestas inmunes innatas y adaptativas, así como en el desarrollo de las enfermedades alérgicas. Rev.cienc.biomed. 2015;6(2):319-332

\section{PALABRAS CLAVE}

Vitamina D; Alergia; Asma; Rinitis alérgica; Dermatitis atópica.

\section{SUMMARY}

Introduction: vitamin $\mathrm{D}$ is one of the most pleiotropic molecules. It is very important in calcium metabolism, pulmonary health and in the immune system. Epidemiological studies have linked vitamin D deficiency with asthma and atopic dermatitis. In addition, some genetic studies including genome scan report association between vitamin $D$ receptor (VDR) and asthma.

\footnotetext{
1 BLc, MSc. Investigadora, Instituto de Investigaciones Inmunológicas. Universidad de Cartagena. Cartagena. Colombia.
}

2 Médico. Profesor Instituto de Investigaciones Inmunológicas. Universidad de Cartagena. Cartagena. Colombia. 
Objective: to identify the role of vitamin D in immune responses and allergic diseases. Methods: electronic search was carried out in the databases, PubMed, Science Direct, Protein Data Bank, NCBI, Blackwell Synergy Wiley Online Library.

Results: 120 articles were selected for full review and 77 and 2 abstracts of them were chosen.

Conclusion: epidemiological and genetics studies have linked vitamin $D$ and its receptor (VDR) with the development of allergic diseases. This evidence is extensive and sometimes contradictory. The apparent contradiction may be explained by the differential recruitment of coactivators RV-VDR-RXR complex. However, experimental studies in vitro and in vivo show that vitamin $\mathrm{D}$ has a modulatory effect on various types of cells of the innate and adaptive immune system, as well as the cells involved in the immune response Th1, Th2, Treg and Th17, concluding that this vitamin plays a key role on innate and adaptive immune system and in the development of allergic diseases. Rev.cienc.biomed. 2015;6(2):319-332

\section{KEYWORDS}

Vitamin D; Allergy; Asthma; Allergic rhinitis; Atopic dermatitis.

\section{INTRODUCCIÓN}

La vitamina $D$ es una de las moléculas más pleiotrópicas, además de los bien conocidos efectos que ejerce sobre el metabolismo del calcio, también se ha podido establecer que influye de manera importante en la salud pulmonar y en el sistema inmune $(1,2)$. Estudios epidemiológicos han relacionado la insuficiencia de esta vitamina con el asma, la dermatitis atópica y los niveles de IgE (3-7). Así mismo, algunos estudios genéticos que incluyen hasta el rastreo genómico reportan una asociación entre el receptor de la vitamina $D(V D R)$ y el asma (8-11).

Las enfermedades alérgicas como el asma $(A A)$, la rinitis (RA) y la dermatitis atópica (DA) son un problema de salud mundial, cuya prevalencia ha aumentado en los últimos años (12-15). Estas enfermedades son consideradas complejas ya que en ellas intervienen factores genéticos y ambientales. Además, son responsables de una disminución notable en la calidad de vida de las personas que las padecen y generan enormes costos a los servicios de salud de algunos países por ser en su mayoría crónicas y requerir una medicación constante (16-18).

Aún no está definido cuáles son las principales causas del incremento en la prevalencia de las enfermedades alérgicas. Existen varias hipótesis entre las que se destacan la de la higiene propuesta por Strachan et al. hace más de dos décadas, en ella se propone que una disminución en la exposición a microrga- nismos es la causa principal del incremento en la prevalencia de la rinitis alérgica en Reino Unido (19).

También se ha responsabilizado a los cambios en el estilo de vida de muchas poblaciones que, pasaron de vivir en ambientes rurales a ambientes urbanos con cambios que incluían nuevos patrones dietarios $(20,21)$. Esto último planteó varias hipótesis entre las que cabe mencionar: la de los ácidos grasos N-PUFAS, la vitamina D, los antioxidantes, la dieta mediterránea, la del menor consumo de frutas y vegetales al igual que la de los pescados (22-25).

Estudios epidemiológicos y experimentales postulan a la vitamina $D$ como responsable al menos en parte del incremento en la prevalencia de las enfermedades alérgicas como el asma $(26,27)$. Por otro lado, algunos estudios genéticos han reportado asociaciones entre el receptor de la vitamina $D$ (VDR) y el asma (8-10). De igual manera, algunos escaneos genómicos en individuos con asma han detectado ligamiento genético en el brazo largo del cromosoma 12, el cual se ha relacionado al menos en parte con la susceptibilidad a padecer esta enfermedad $(11,28)$.

En un modelo murino de asma alérgica experimental concomitantemente con la inmunoterapia se administró vitamina $D$, se observó una disminución significativa de la hiperreactividad de vías aéreas y los parámetros de la inflamación, mostrando un incremento de citoquinas reguladoras como IL-10. Lo 
anterior demuestra el papel potenciador de la inmunoterapia de la vitamina D (29).

\section{MATERIALES Y MÉTODOS}

Tipo de estudio: revisión descriptiva en la cual se incluyeron articulos epidemiológicos, experimentales, genómicos y de revisión.

Tipo de participantes: artículos publicados con alto impacto en el tema, en los que se demuestra claramente el efecto de la vitamina $D$ sobre las distintas células del sistema inmune y además, aquellos que establecen algún papel fundamental de la vitamina $D$ en las enfermedades alérgicas.

Estrategias de búsqueda bibliográfica: se realizó una búsqueda electrónica en las bases de datos Pubmed, ScienceDirect, Protein Data Bank, NCBI, Blackwell Synergy Wiley Online Library, el idioma considerado para esta búsqueda fue exclusivamente el inglés.

Términos claves: se estableció la búsqueda con las palabras claves vitamin D, 1 a 25 -dihydroxy vitamin D3, 25-hydroxy vitamin D3, allergy, asthma, rhinitis, atopic dermatitis, genomic scan, vitamin $\mathrm{D}$ receptor (VDR), vitamin $D$ gene regulation, monocytes, T-lymphocytes, B-lymphocytes, dendritic cells and neutrophils.

Metodos de revisión: inicialemnte se escogieron los articulos por su nombre y resumen, luego se examinó el texto completo de los documentos previamente escogidos de las bases de datos. Se selecccionaron aquellos en los que se demostrara un efecto de la vitamina $D$ sobre las celulas del sistema inmunológico y sobre las enfermedades alérgicas, además, se describió el metabolismo y el mecanismo de acción de la vitamina $D$. La revisión de los artículos fue realizada de forma independiente por los autores.

\section{RESULTADOS}

Se escogieron un total de 120 artículos, de los cuales 77 eran artículos completos y 2 resúmenes de nuestro grupo de investigación que fueron utilizados para esta revisión.
La vitamina $D$ proviene de dos fuentes: la exposición de la piel a los rayos ultravioleta (UV) y la ingesta proveniente de la dieta. Las fuentes dietéticas incluyen el pescado, el hígado, la yema de huevo y los suplementos dietarios. Como muy pocos alimentos contienen vitamina $\mathrm{D}$, la exposición al sol es el principal determinante de los niveles de vitamina $D$ en los seres humanos. En esta revisión se describirá el metabolismo y los mecanismos de acción de la vitamina $D$, el papel de esta en las respuestas inmunes innata y adaptativa, así como en el desarrollo de enfermedades alérgicas como el asma, la rinitis y la dermatitis atópica.

\section{METABOLISMO Y MECANISMO DE AC- CIÓN DE LA VITAMINA D}

La síntesis de la vitamina D3 se produce en la piel, en esta se convierte en pre-vitamina D3 en respuesta a la exposición a la luz UV (Iongitud de onda 290-315). La vitamina D3 obtenida a partir de pre-vitamina D3 en la piel o por la absorción intestinal de componentes de la dieta se une a la proteína de unión a vitamina D (DBP) en la circulación (30).

La DBP es una proteína multifuncional altamente polimórfica y que se produce en grandes cantidades en el hígado y que se encarga de transportar la vitamina $D$ al mismo órgano (30). Aquí la vitamina D3 es hidrolizada por 25-hidroxilasa (CYP2R1) convirtiéndose en $25(\mathrm{OH})$ D3, la cual posteriormente es nuevamente hidrolizada en el riñón por la 1-alfa-hidroxilasa (CYP27B1), lo que genera la hormona activa 1,25 (OH $)_{2}$ D3 (31).

La $25(\mathrm{OH})_{2}$ D3 es la principal forma circulante de la vitamina $D$, tiene una vida media de aproximadamente dos semanas. Este metabolito es considerado como el principal indicador del estado de la vitamina D. En personas sanas, $25(\mathrm{OH})_{2}$ D3 está presente en el suero a concentraciones que se encuentran en el rango de entre 30 a $50 \mathrm{ng} / \mathrm{ml}$. Los individuos con niveles séricos $<30 \mathrm{ng} /$ $\mathrm{ml}$ presentan insuficiencia de esta, mientras que se proponen niveles $<15 \mathrm{ng} / \mathrm{ml}$ para definir la deficiencia de esta vitamina.

Es importante destacar que, como es una vitamina soluble en grasa, $25(\mathrm{OH})_{2}$ D3 puede 
también ser almacenada en los músculos y en el tejido adiposo (32). Además, algunas células del sistema inmune como los linfocitos $T$, linfocitos $B$, macrófagos y algunas subpoblaciones de células dendríticas poseen la maquinaria enzimática para producir la 1,25-dihidroxivitamina D3, (Figura 1). Por último, la 24-hidroxilasa cataliza la conversión de este último metabolito en ácido calcitroico, en el intestino y en los riñones, para luego ser excretado por la bilis $(33,34)$.

La 1,25-dihidroxi-vitamina D3 ejerce sus acciones a través del VDR presente en las células blanco. Este receptor nuclear que pertenece a la superfamilia de receptores de hormonas esteroideas, posee dos dominios discretos: el $\mathrm{N}$ - terminal de unión al ADN (DBD) y el C-terminal de unión a los ligandos (LDB), su estructura cristalográfica fue dilucidada por Kashiwagi, H. y cols. y se encuentra depositada en Protein Data Bank (PDB; 3WOA), (Figura 2). La alta afinidad de unión del dominio LBD a la $1,25(\mathrm{OH})_{2} \mathrm{D} 3$ induce la heterodimerización del VDR con el receptor del ácido retinoico $X$ (Retinoic $X$ Receptor, $R X R)$, para la activación de genes dianas de la vitamina $\mathrm{D}$.

Una vez formado el complejo heterodimérico $1,25(\mathrm{OH})_{2} \mathrm{D} 3-\mathrm{VDR}-\mathrm{RXR}$, este interactúa con secuencias de ADN específicas, denominadas elementos respondedores a la vitamina $D$ (VDREs), presentes en el promotor de los genes blanco reprimiendo o activando la transcripción. EI VDRE se caracteriza por te- ner repeticiones directas de dos motivos de unión central hexaméricas (siendo preferencialmente AGTTCA) espaciadas por tres nucleótidos (35). La unión del VDR al elemento respondedor (VDRE) recluta coactivadores y enzimas que activan la acetilación de las histonas, causando cambios estructurales en la cromatina, por lo tanto, facilitan la transcripción de genes (36).

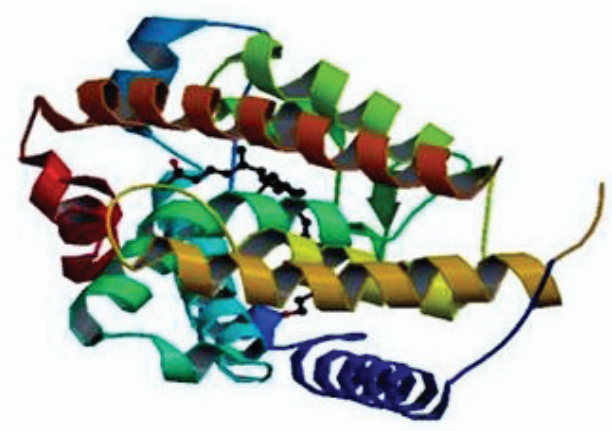

Figura 2. Imagen 3D del VDR. PDB 3WOA.

La actividad transcripcional de los genes de receptores nucleares y otros factores de transcripción es actualmente entendida como un proceso de múltiples pasos que se inicia en la región promotora de los genes expresados. El TFIIB y varios factores asociados a proteína de unión a TATA, así como los coactivadores p160 conocidos como activadores de hormonas esteroideas (SRC- I, SRC- 2, y el SRC- 3), tienen actividad de acetilación de histonas (HAT) y ha sido reportado que se involucran en la transcripción mediada por el VDR (37).

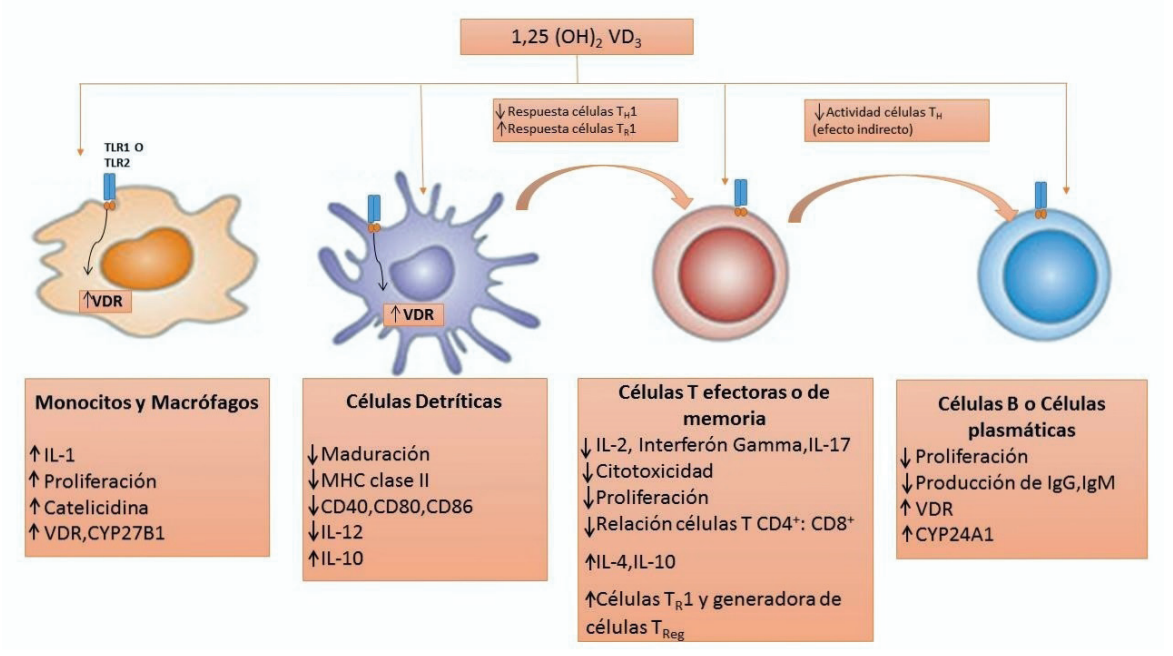

Figura 1. Efecto de la vitamina D en las células del sistema inmune.
En estudios recientes se ha demostrado la cooperatividad entre histonas metiltransferasas y coactivadores SRC, lo que los lleva a desempeñar un papel fundamental en la activación transcripcional en diversos tipos de células, mediante el reclutamiento diferencial de coactivadores. El reclutamiento de la RNA polimerasa II mediado por VDR está determinado por un complejo coacti- 
vador conocido como DRIP (proteína que interactúa con el VDR) este complejo no posee actividad HAT.

Además, se ha sugerido que el complejo coactivador SRC/CREB (CBP) es reclutado primero por el remodelamiento de la cromatina seguido por el reclutamiento de la maquinaria de transcripción por el complejo $\operatorname{DRIP}(38,39)$. Adicionalmente, un número de factores de transcripción promotor específicos, incluidas las proteínas $\beta$ y $\delta$ de unión a los potenciadores YY1 y CCAAT han sido reportados como moduladores de la transcripción mediada por VDR. Todo lo anterior sugiere que las funciones activadoras de promotores de funciones específicas del VDR pueden ser mediadas por reclutamiento diferencial de coactivadores, es decir, la vitamina D3 puede regular la vía de señalización del NF-kB mediante la unión con el VDR y el incremento de la expresión de la proteína inhibitoria IKBa (40).

\section{VITAMINA D Y RESPUESTA INMUNE}

Existe un gran consenso en que la vitamina $D$ juega un papel importante en la regulación de la respuesta inmune. El receptor de la vitamina $D(V D R)$ y las enzimas capaces de convertir $25(\mathrm{OH})$ vitamina-D a $1,25(\mathrm{OH})_{2}$ vitamina-D3 se han detectado en células del sistema inmune de los linajes mieloide y linfoide, tanto en reposo como en activación y en los LT-CD4+ y CD8+ activados, linfocitos $B$, neutrófilos y células presentadoras de antígeno (CPA) como los macrófagos y las células dendríticas (CD) $(41,42)$. Lo anterior permitió postular la hipótesis de que la producción local de vitamina D podría ejercer un efecto modulador sobre estas células.

En varios estudios se ha podido establecer que la vitamina D3 modula la actividad de monocitos/macrófagos y células dendríticas. En estas últimas inhibe su diferenciación y maduración, reduce la expresión de moléculas del complejo mayor de histocompatibilidad clase II, del CD40, CD80 y del CD86, además, inhibe la secreción de IL-1, IL-6, TNF-a e IL-12 $(43,44)$.

En un modelo in vitro Penna G., y cols. demostraron que la vitamina D3 en presencia de CPA disminuye la producción de INF-Y sin afectar la producción de citoquinas Th2 (45) y la administración in vivo de vitamina D incrementa la expresión y producción de IL-4 con una disminución de los niveles de INF-Y (46). Esta inhibición se podría deber a la disminución de la producción de IL-12 por parte de las CPA luego del tratamiento con la vitamina D (47). Del mismo modo, Boonstra A. y cols., establecieron que la vitamina D3 podía también ejercer su efecto sobre la polarización de la respuesta hacia el perfil Th2 mediante la acción directa sobre las células T CD4+ (48).

[A] VITAMINA D Y RESPUESTA INMUNE INNATA: la respuesta inmune innata es un mecanismo inespecífico de defensa de suma importancia en el organismo, responde de manera rápida contra microorganismos patógenos. La activación de los receptores Toll (TLR) desencadena la expresión de genes que codifican para péptidos antimicrobianos como la catelicidina y reducen la viabilidad de Micobacterium tuberculosis en monocitos y macrófagos humanos, mas no en células dendríticas (DC) (49). En monocitos humanos Liu, y cols., establecieron que la activación del heterodímero TLR2/TLR1 por parte de micobacterias incluyendo el bacilo tuberculoso, producía el incremento en la expresión de catelicidina debido al aumento en la activación de los genes del VDR y la 1-hidroxilasa de vitamina D (Cyp27B1) en monocitos y macrófagos. Además, estos autores estudiaron la funcionalidad del VDR en estas mismas células demostrando que su activación inducía al menos a la producción de un péptido antimicrobiano.

En este mismo estudio y reforzando la hipótesis que individuos de raza negra tienen mayor susceptibilidad a la tuberculosis debido a menores niveles de vitamina $D$, se observó que el suero de voluntarios de raza negra indujo una menor expresión del gen de la catelicidina en los cultivos de monocitos en comparación con el suero de individuos caucásicos (50).

Chun RF y cols. analizaron la biodisponibilidad de la 25(OH)D en monocitos de ratones knockout de DPB y ratones heterocigóticos para este gen, establecieron que algunas 
de las variantes genéticas de esta proteína con mayor afinidad (Gc1F-1F) por las formas de la vitamina $D$ mostraban mayor capacidad de inhibir la producción de catelicidina en los monocitos que aquellas variantes de baja afinidad (Gc21F-1S y Gc2-2) (51). Estudios en individuos con dermatitis atópica, Hata y cols. demostraron que la suplementación con vitamina D oral (Colecalciferol) incrementó de manera significativa los niveles de catelicidina en la piel de estos individuos, sugiriendo que esta podría jugar un papel importante en la prevención de infecciones cutáneas en estos pacientes (52).

Los neutrófilos y sus péptidos microbicidas son otro componente importante de la respuesta inmune innata, especialmente por su capacidad para matar bacterias de manera rápida. Wang TT. y cols. en un modelo in vitro con neutrófilos humanos demostraron que la 1,25 dihidroxi vitamina D3 sola o combinada con LPS induce la expresión del gen del péptido antimicrobiano (53).

Adicionalmente, Karlsson y col., demostraron que los pacientes con neutropenia congénita severa (NCS) tenían una disminución del péptido antimicrobiano LL-37 y una mayor susceptibilidad a las infecciones. En ese mismo artículo los autores demostraron que la vitamina D3 era capaz de inducir un incremento en la expresión y producción del péptido LL-37, mejorando así la capacidad de los neutrófilos de estos pacientes para matar bacterias (54).

[B] VITAMINA D Y LA RESPUESTA INMUNE ADAPTATIVA: la respuesta inmune adaptativa es generalmente definida por los linfocitos T y B y su capacidad para producir citoquinas e inmunoglobulinas respectivamente, además, para combatir específicamente la fuente de los antígenos que son presentados ante estos por células del sistema inmune innato. Tanto los LTh1 como los Th2 están involucrados en la patogénesis de diversas enfermedades entre las que se destacan las autoinmunes, para los primeros y las alergias en los segundos. Por lo tanto, el estudio de moléculas como la vitamina D3 que puedan modular una u otra respuesta tendrá importantes implicaciones en el tratamiento de estas enfermedades.
[C] EFECTO DE LA VITAMINA D EN LOS LINFOCITOS TH1 Y TH2: está bien establecido que el VDR se expresa en casi todas las células del sistema inmune como los LT CD4+, CD8+ activados, neutrófilos y APCs como los macrófagos y las células dendríticas. Las señales de activación del sistema inmune son las que inducen la expresión del VDR en estas células. Mientras que los LT vírgenes expresan muy bajos niveles del VDR, este receptor es expresado abundantemente en los LT activados. En contraste con lo que sucede en las APC, su maduración va acompañada de una disminución en la expresión del VDR (55).

La mayoría de los genes de las citoquinas que se expresan durante los procesos inflamatorios mediados por LT CD4+, Th1 o Th2, son regulados por el factor de transcripción NFkB (56). Es bien conocido el potente efecto inhibidor de la vitamina D3 en la expresión de este factor de transcripción en las células dendríticas y linfocitos T. En las primeras, previene su maduración incrementando su potencial tolerogénico y en los LT inhibe, especialmente, la producción de citoquinas del perfil Th1 e incrementa la producción de IL-4 (57).

Sin embargo, los estudios que demuestran el efecto directo de la vitamina $D$ sobre los linfocitos $\mathrm{T}$ o algunas de sus subpoblaciones son escasos. Rigby y cols., por medio de un modelo in vitro con PBMC humanas demostraron que la proliferación de linfocitos $T$ y la producción de IL-2 eran significativamente menores cuando las células eran estimuladas con PHA y expuestas a 1,25-dihidroxivitamina D3 sugiriendo que el efecto podría ser a través de la baja regulación de expresión del receptor de IL-2 (58).

No obstante, otros investigadores han demostrado que la vitamina $D$ puede tener efecto inmunomodulador sobre los linfocitos $T$ actuando sobre la expresión de un grupo de genes relacionados con su metabolismo. De la misma manera, Baeke F. et al., analizaron la expresión y la cinética de algunos genes relacionados con la vitamina D (24 hidroxilasa y 1 alfa hidroxilasa, VDR) y además, analizaron si la activación de las vías de señalización de células T eran depen- 
dientes del tipo de estímulo (anti-CD3, anti CD28, PHA, PMA, ionomicina, rIL-12, rIL-2). Mediante un modelo in vitro con el que evaluaron estos efectos sobre poblaciones de linfocitos $T$ activados, encontraron que la adición de la 1,25 dihidroxivitamina $D$ activa efectivamente la señalización del VDR, como lo demuestra la inducción de la enzima 24 hidroxilasa, solo cuando los linfocitos $T$ expresan altos niveles de VDR. Este incremento en la señalización a través del VDR se correlaciona con una fuerte inhibición de las citoquinas IFN- $\mathrm{Y}, \mathrm{IL}-10$ y la modulación de la expresión de receptores para el residenciamiento como el CCR10 y el CLA. Sobre la base de estos resultados se observó que el tratamiento con 1,25 dihidroxivitamina $D$ es un factor crítico para la eficiente activación de las vías de señalización del VDR, concluyendo que la células $T$ son blancos directos de la 1,25 dihidroxivitamina D3 (57).

Staeva-Vieria TP, y cols., en un modelo in vitro con células $T$ vírgenes de ratón pudieron establecer el efecto inhibitorio sobre las citoquinas Th1 (INFY) y las Th2 (IL-4) durante el proceso de polarización de la respuesta. Sumado a ello, demostraron que en LT CD4 + ya diferenciados el efecto inhibitorio era menor sobre el INFY y nulo sobre la producción IL-4. Como mecanismo de esta inhibición los autores propusieron que se debía al efecto inhibitorio de la transcripción de los genes de las citoquinas por parte del VDR (59).

En la mayoría de los estudios que evalúan el efecto de la vitamina D3 en la respuesta inmune adaptativa, no queda claro si el efecto sobre la expresión de citoquinas en los LT (Th1 o Th2) no estaría involucrando a otras poblaciones celulares como las APC. Con el fin de demostrar el efecto directo de la vitamina D3 sobre los LT, Jeffrey L.E, y cols., utilizando LTs CD4+ CD25- demostraron que la $1,25(\mathrm{OH})_{2}$ D3 inhibe la producción de citoquinas proinflamatorias como el INFY, IL17 e IL-21, sin inhibir la proliferación celular; en contraste con el efecto inductor de la expresión del CTLA-4 y Foxp3, esto último en presencia de la IL-2. En este mismo estudio los autores demostraron que los LT tratados con vitamina D3 poseía capacidad de inhibir la respuesta proliferativa de LT normales, indicando que estas células juegan un papel regulador en la respuesta inmune adaptativa (60).

En otro estudio, Boonstra A. y cols., mediante un modelo murino usando LT CD4+Mel14* derivados de ratones con fondo genético BALB/C y C57BL/6 demostraron que, la vitamina D3 afectaba la polarización de los LTh al inhibir la producción de citoquinas Th1 (INFY) y aumentar las Th2 (IL-4, IL-5 e IL10), tanto en cultivos de LT estimulados con APCs como en LT solos y estimulados con anti-CD3 y anti-CD28, lo que indicaba que los LT CD4+ también pueden ser blanco directo de la vitamina D3. El incremento de la respuesta Th2 fue observado en ambas cepas de ratón, BALB/c y C57BL/6 y correlacionaba con la expresión de los factores de transcripción específicos de LTh2 c-maf y GATA-3. Además, se estableció que el efecto directo de la vitamina D3 sobre los Th2 era dependiente de la IL-4 ya que este desaparecía al utilizar anti-IL-4 (48).

[D] EFECTO DE LA VITAMINA D EN LAS CÉLULAS TH17: las células Th17 son un linaje de LT CD4+ que producen IL-17 y a las que se las ha implicado en la patogénesis de algunas enfermedades autoinmunes como la esclerosis múltiple, el lupus eritematoso sistémico, la artritis reumatoide y en enfermedades atópicas como la DA y algunos tipos de asma resistente a los esteroides (61).

En un estudio Joshi S y cols., utilizando células $T$ humanas y de ratón, demostraron que la vitamina D3 inhibía la producción de IL-17 en ambos tipos de células. Se observó que la administración de vitamina D3 a ratones con encefalomielitis autoinmune experimental (EAE), disminuía la parálisis en los animales y reducía los niveles de LT CD4+ secretores de Il-17 en la periferia y el sistema nervioso central. También establecieron que el mecanismo represivo de la vitamina D3 involucraba el bloqueo del factor nuclear de activación de los LT (NFAT), el reclutamiento de deacetilasa de histonas (HDAC) y el secuestramiento del factor de transcripción 1 relacionado con Runt (Runx1) (62).

El estudio de Nanzer A. y cols., con PBMC de pacientes asmáticos sensibles y resistentes a los esteroides, demostraron que la 1a,25- 
dihidroxivitamina D3 tiene la capacidad de inhibir la producción de citoquinas Th17 (IL -17A e IL -22), independientemente de su respuesta clínica a los esteroides, además, propusieron un posible mecanismo para la inhibición de la síntesis de IL -17A en las células $T$, por intermedio de la ectonucleotidase CD39, que se expresa en las células $T$ de memoria y del Foxp3 en las células Treg (61).

[E] EFECTO DE LA VITAMINA D EN LOS LINFOCITOS B: los LB son las células productoras de anticuerpos responsables de los mecanismos claves de la inmunidad humoral. El control de la producción de anticuerpos por estas células es ejercido principalmente por los LT y sus citoquinas. Las cuales inducen el cambio de isotipos en los genes de las inmunoglobulinas para la producción de los diferentes isotipos de anticuerpos como la $\mathrm{IgE}$, entre otros.

Diversos estudios han relacionado los niveles bajos de vitamina $D$ con enfermedades autoinmunes como el LES y con las alergias. Aunque la causa exacta de las enfermedades autoinmunes no se conoce, la depleción de LB ha demostrado ser una terapia efectiva y eficiente para el control de las mismas, sugiriendo que los LB y los anticuerpos juegan un papel importante en la patogénesis de estas enfermedades (63).

Estudios realizados en LB humanos activados con anti-CD40 + IL-4 y tratados con vitamina $D$ demostraron que esta molécula inhibía la producción de IgE sin afectar la proliferación celular ni la síntesis de otros isotipos de inmunoglobulinas. Al evaluar la producción de IL- 6 en estas células se observó que hubo un incremento en la producción de esta citoquina, estos datos sugieren que la inhibición de la síntesis de IgE por vitamina $D$ no se produce a través de la inhibición de la IL-6. Adicionalmente, se observó una inhibición en la expresión del CD23 en los cultivos de LB cuando eran estimulados solamente con IL4 y un ligero incremento en la inhibición cuando se adicionaba anti-CD40. Dada la importancia que juega el CD23 y la IL-6 en la síntesis de la IgE, los autores sugieren que la vitamina $D$ podría tener un papel importante como agente terapéutico en las enfermedades alérgicas (64).

\section{VITAMINA D $Y$ LAS ENFERMEDADES ALÉRGICAS}

Diversos estudios epidemiológicos han demostrado una relación entre la deficiencia e insuficiencia de vitamina D con el asma y otras enfermedades alérgicas (27-65). Algunos estudios grandes de corte transversal han mostrado una asociación entre los niveles bajos de vitamina $D$ y una función pulmonar reducida en adolescentes $(66,67)$.

Osborne y Ukoumunne, determinaron las prevalencias de las enfermedades alérgicas en tres latitudes de Australia (norte o más cercana a la línea ecuatorial, central y sur) encontrando que el reporte de eczema y de alergia alimenticia se relacionaba gradualmente con estar ubicado en la región sur, los autores sugieren que una menor exposición a la radiación UV y la obtención de vitamina D podría determinar dicho riesgo (68). Una dieta rica en vitamina $D$ durante el embarazo se relaciona con menor probabilidad de enfermedad respiratoria en los niños (69).

Por ser la exposición a la luz solar la principal fuente de producción de vitamina $D$ de muchos individuos y las dietas cada vez más pobres en este micronutriente, se postula que los estilos de vida más modernos, con mucho tiempo intradomiciliario y con poca exposición a la luz solar han jugado un papel muy importante en el aumento de la prevalencia de las enfermedades alérgicas.

[A] VITAMINA D, ASMA Y RINITIS ALÉRGICA: el asma y la rinitis alérgica son enfermedades inflamatorias en las que participan y juegan un papel central en su patogénesis los linfocitos Th2. Estos por intermedio de sus citoquinas como la IL-4, IL-5 e IL-13 inducen la síntesis de IgE, la eosinófilia y la producción de moco por parte del epitelio de las vías respiratorias, sin embargo en el caso del asma incrementan la reactividad de las vías aéreas (70).

Tolppanen $y$ cols., reportaron recientemente que altos niveles de 25-hidroxi-vitamina $D_{2}$ y 25-hidroxivitamina- $D_{3}$ en una cohorte de más de 3.300 niños con edad promedio de 9.8 años del sudeste de Inglaterra se asociaron con un mayor riesgo de sibilancias y 
dermatitis (71); en contraste con un estudio realizado por Kolokotroni y cols., en una cohorte de 5.384 adolescentes griegos con edades de entre 16 a 17 años que presentaban una tendencia a tener niveles más bajos de vitamina $D$ en asmáticos comparados con los no asmáticos (72).

Erkkola y cols., encontraron una asociación protectora entre el consumo de vitamina $D$ de 1.669 madres durante el embarazo y el desarrollo de síntomas de asma y/o rinitis alérgica de sus hijos a los 5 años, la cual se mantuvo incluso después del ajuste por variables de confusión y otros componentes de la dieta que también pudiesen explicar este hallazgo (73). Una revisión sistemática reciente encontró que 5 de 6 estudios prospectivos de cohortes de nacimiento, reportaban una asociación inversa entre la ingesta de vitamina $D$ durante el embarazo y la probabilidad de desarrollar sibilancias en los hijos (69).

Los individuos de raza negra tienen una menor producción de vitamina $D$ luego de exponerse a los rayos UV. En un estudio en niños afroamericanos con asma, Freishtat y cols., encontraron un alto porcentaje de deficiencia $(54 \%,<20 \mathrm{ng} / \mathrm{mL}$ ) e insuficiencia $(86 \%,<30 \mathrm{ng} / \mathrm{mL})$ de 25-hidroxi-vitamina D (74). En Latinoamérica, otro estudio que incluía 616 costarricenses muestra que el $28 \%$ presentaron niveles insuficientes de vitamina $\mathrm{D}$, a su vez, describieron una relación inversa de esta con los niveles de IgE total y específica a ácaros, número de eosinófilos en sangre periférica y el tamaño de la pápula en la prueba cutánea. De igual forma se asoció negativamente con una mayor respuesta al reto con metacolina y con una menor probabilidad de hospitalización en el año previo (75). De esta manera la evidencia epidemiológica parece indicar que la vitamina D participa en los procesos fisiopatológicos que conllevan a las enfermedades alérgicas. A pesar de que algunos resultados son contradictorios, esto podría deberse a un efecto "U-shaped" donde los extremos de las concentraciones de la vitamina D se asocian con el fenotipo alérgico como con los niveles elevados de IgE, sugiriendo un efecto modulador más que supresor de la respuesta inmune de tipo alérgico $(6,7)$.
[B] VITAMINA D Y DERMATITIS ATÓPICA (DA): la dermatitis atópica (DA) es una enfermedad inflamatoria de la piel en la que hay un disfunción en las barreras epidérmica e inmunológica. Estos pacientes poseen un defecto en la permeabilidad de la barrera cutánea así como en la capacidad antimicrobiana del estrato corneo. La barrera cutánea compuesta por lípidos hidrofóbicos que percloran el ambiente extracelular del estrato corneo y previenen la pérdida de agua. Sumado a lo anterior, se observa hiperactividad de proteasas de serina secundaria a defectos genéticos como el de la filagrina (FLG) y a estímulos ambientales que promueven la reducción de la hidratación y de la producción de lípidos extracelulares en el estrato corneo, facilitando la introducción de antígenos y la promoción de la inflamación (76). Existen estudios que demuestran que mutaciones funcionales en el gen de la FLG (Localizada en el Cr 1q21) se asocian con DA (77).

Los péptidos antimicrobianos como la catelicidina son secretados en la superficie de la piel y tienen un papel importante en la primera línea de defensa cutánea. Estos se liberan en respuesta a la activación de los receptores Toll (TLR) presentes en la superficie celular de los queratinocitos y células cebadas. En la DA se ha observado una disminución en la expresión de la catelicidina en comparación con lesiones psoriásicas. Lo cual explica las diferencias en la susceptibilidad al desarrollo de infecciones observadas entre los pacientes con $A D$ y los que padecen psoriasis. Se ha demostrado que la vitamina D tiene un papel importante en la expresión de la catelicidina de la piel y de los monocitos y macrófagos aumentando su capacidad microbicida, gracias al incremento en la expresión del VDR y de la enzima 1,25 hidroxilasa en estas células (50).

Al igual que con muchas de las enfermedades atópicas, hay datos conflictivos que rodean el efecto que tiene la vitamina $D$ en el desarrollo de las enfermedades alérgicas de la piel. Gale, C. et al., en un estudio prospectivo en el que se reclutaron 596 mujeres embarazadas y se analizaron 466 (78\%) niños al nacer, 440 (74\%) a los 9 meses y $178(30 \%)$ a la edad de 9 años, observaron que los niños cuyas madres tenían concentraciones de $25(\mathrm{OH}) \mathrm{D}$ 
mayores de $75 \mathrm{nmol} / \mathrm{L}$ tenían un mayor riesgo de desarrollar eczema a los 9 meses (OR 3,26 , IC del $95 \% 1.15$ a 9.29, $\mathrm{p}=0.025$ ) y asma a los 9 años (OR 5.40, IC del $95 \%$, 1.09-26.65, $p=0.038$ ), en comparación con los niños cuyas madres tenían concentraciones menores de $30 \mathrm{nmol} / \mathrm{I}$ (78).

Oren, E. et al., en un estudio de casos y controles en 290 pacientes obesos observó un incremento de cinco veces la probabilidad de padecer DA en los sujetos con deficiencia de vitamina $D$ comparados con el grupo con suficiencia (67). En otro estudio realizado en una población coreana donde evaluaron la relación de los niveles de vitamina $D$ con la atopías en 15212 individuos adultos de esta población, encontraron un mayor riesgo de DA en individuos con niveles bajos de vitamina $D$, sin evidencia de relación con asma ni rinitis alérgica (79).

\section{CONCLUSIÓN}

Los estudios epidemiológicos y de genética han relacionado a la vitamina $D$ y su receptor (VDR) con el desarrollo de las enferme- dades alérgicas. Esta evidencia es extensa y en ocasiones contradictoria, esta contradicción se podría explicar por el reclutamiento diferencial de coactivadores del complejo VD-VDR-RXR y a diferencias poblacionales en los niveles de la proteína transportadora de la vitamina $\mathrm{D}(\mathrm{DBP})$.

Sin embargo, la mayoría de los estudios experimentales in vitro e in vivo demuestran que la vitamina $D$ ejerce un efecto modulador sobre diversos tipos de células del sistema inmune innato y adaptativo, asimismo sobre las células involucradas en la respuesta inmune del tipo Th1, Th2, Treg y Th17, concluyendo que esta vitamina juega un papel fundamental sobre las respuestas inmunes innata y adaptativa, así como en el desarrollo de las enfermedades alérgicas.

CONFLICTOS DE INTERESES: ninguno que declarar.

FINANCIACIÓN: recursos propios del grupo de investigación Alergología Experimental e Inmunogenética Col 000363. Universidad de Cartagena-Colombia.

\section{REFERENCIAS BIBLIOGRÀFICAS}

1. Gupta A, Bush A, Hawrylowicz C, Saglani S. Vitamin D and asthma in children. Paediatr Respir Rev [Internet]. Elsevier Ltd; 2012 Dec [cited 2013 Mar 6];13(4):236-43; quiz 243. Available from: http://www.ncbi.nlm.nih.gov/pubmed/23069123.

2. Muehleisen B, Gallo RL. Vitamin D in allergic disease: Shedding light on a complex problem. J Allergy Clin Immunol [Internet]. Elsevier Ltd; 2013 Feb [cited 2013 Mar 6];131(2):324-9. Available from: http://www.ncbi.nlm.nih.gov/pubmed/23374263.

3. Bäck O, Blomquist HKS, Hernell O, Stenberg B. Does vitamin D intake during infancy promote the development of atopic allergy? Acta Derm Venereol [Internet]. 2009 Jan [cited 2013 Mar 6];89(1):28-32. Available from: http://www.ncbi.nlm.nih.gov/pubmed/19197538.

4. Maria I, Costa C. Dermatite atópica e vitamina D : fatos e controvérsias. 2013;88(6):945-53.

5. Samochocki Z, Bogaczewicz J, Jeziorkowska R, Sysa-Jędrzejowska A, Glińska O, Karczmarewicz $E$, et al. Vitamin $D$ effects in atopic dermatitis. J Am Acad Dermatol [Internet]. 2013 Aug [cited 2014 May 24];69(2):238-44. Available from: http://www.ncbi.nlm.nih.gov/pubmed/23643343.

6. Hyppönen E, Berry DJ, Wjst M, Power C. Serum 25-hydroxyvitamin D and IgE - a significant but nonlinear relationship. Allergy [Internet]. 2009 Apr [cited 2013 Mar 6];64(4):613-20. Available from: http://www.ncbi.nlm.nih.gov/pubmed/19154546.

7. Munoz C, Meza C, Hernandez LDC, Egea E, Marrugo JA. Immunology U of C, Artagena. Influence of vitamin $D$ in IgE levels in rural and urban Colombian African descendent children. Allergy Eur J Allergy Clin Immunol. 2013;68:653-4.

8. Poon AH, Laprise C, Lemire M, Montpetit A, Sinnett D, Schurr E, et al. Association of vitamin D receptor genetic variants with susceptibility to asthma and atopy. Am J Respir Crit Care Med [Internet]. 2004 Nov 1 [cited 2014 Jun 4];170(9):967-73. Available from: http://www.ncbi. nlm.nih.gov/pubmed/15282199.

9. Raby B, Lazarus R, Silverman EK, Lake S, Lange C, Wjst M, et al. Association of vitamin D receptor gene polymorphisms with childhood and adult asthma. Am J Respir Crit Care Med [Internet]. 2004 Nov 15 [cited 2014 Jun 4];170(10):1057-65. Available from: http://www. ncbi.nlm.nih.gov/pubmed/15282200. 
10. Ismail MF, Elnady HG, Fouda EM. Genetic variants in vitamin D pathway in Egyptian asthmatic children: a pilot study. Hum Immunol [Internet]. American Society for Histocompatibility and Immunogenetics; 2013 Dec [cited 2014 Jun 18];74(12):1659-64. Available from: http://www. ncbi.nlm.nih.gov/pubmed/24007655.

11. Wjst M, Altmüller J, Braig C, Bahnweg M, André E. A genome-wide linkage scan for 25-OH-D(3) and 1,25-(OH)2-D3 serum levels in asthma families. J Steroid Biochem Mol Biol [Internet]. 2007 Mar [cited 2014 Jun 18];103(3-5):799-802. Available from: http://www.ncbi.nlm.nih. gov/pubmed/17236760.

12. Study TI, Committee S. Worldwide variation in prevalence of symptoms of asthma, allergic rhinoconjunctivitis, and atopic eczema : ISAAC. 1998;351:1225-32.

13. Asher MI, Montefort S, Björkstén B, Lai CKW, Strachan DP, Weiland SK, et al. Worldwide time trends in the prevalence of symptoms of asthma, allergic rhinoconjunctivitis, and eczema in childhood: ISAAC Phases One and Three repeat multicountry cross-sectional surveys. Lancet [Internet]. 2006 Aug 26;368(9537):733-43. Available from: http://www.ncbi.nlm.nih.gov/ pubmed/16935684.

14. Marrugo J, Hernández L, Villalba V. Prevalence of self-reported food allergy in Cartagena ( Colombia ) population. 2008;36(6):320-4.

15. Dennis RJ, Caraballo L, García E, Rojas MX, Rondon Ma, Pérez A, et al. Prevalence of asthma and other allergic conditions in Colombia 2009-2010: a cross-sectional study. BMC Pulm Med [Internet]. BioMed Central Ltd; 2012 Jan [cited 2014 Jun 15];12(1):17. Available from: http://www. pubmedcentral. nih.gov/articlerender.fcgi?artid=3488963\&tool=pmcentrez\&rendertype=abstract

16. Yepes-Nuñez JJ, Gómez-García C, Espinosa-Herrera Y, Cardona-Villa R. Health-related quality of life in children and adults with respiratory allergy in Colombia: prospective study. Allergol Immunopathol (Madr) [Internet]. 2012 [cited 2014 Jun 15];40(6):379-84. Available from: http:// www.ncbi.nlm.nih.gov/pubmed/22316550.

17. Small M, Piercy J, Demoly P, Marsden H. Burden of illness and quality of life in patients being treated for seasonal allergic rhinitis: a cohort survey. Clin Transl Allergy [Internet]. 2013 Jan;3(1):33. Available from: http://www.pubmedcentral.nih.gov/articlerender.fcgi?artid=3852 977\&tool=pmcentrez\&rendertype=abstract.

18. Law AW, Reed SD, Sundy JS, Schulman $\mathrm{K}$ a. Direct costs of allergic rhinitis in the united states: Estimates from the 1996 medical expenditure panel survey. J Allergy Clin Immunol [Internet]. 2003 Feb [cited 2014 Jun 15];111(2):296-300. Available from: http://linkinghub.elsevier.com/ retrieve/pii/S0091674902913308.

19. Strachan DP. household size. Br Med J. 1989;299(November):1259-60.

20. Hooper R, Calvert J, Thompson RL, Deetlefs ME, Burney P. Urban/rural differences in diet and atopy in South Africa. Allergy [Internet]. 2008 Apr [cited 2011 Oct 29];63(4):425-31. Available from: http://www.ncbi.nlm.nih.gov/pubmed/18315730.

21. Marrugo J, Urrego J, Fang L, Hernandez L LL. Influence of rural and urban dietary and lifestyle patterns in atopic diseases in a Colombian African-descent population. J Allergy Clin Immunol. $2011 ; 8334$.

22. Devereux G, Seaton A. Diet as a risk factor for atopy and asthma. J Allergy Clin Immunol [Internet]. 2005 Jun [cited 2011 Aug 14];115(6):1109-17; quiz 1118. Available from: http://www. ncbi.nlm.nih.gov/pubmed/15940119.

23. Allan K, Devereux G. Diet and asthma: nutrition implications from prevention to treatment. J Am Diet Assoc [Internet]. Elsevier Inc.; $2011 \mathrm{Feb}$ [cited $2011 \mathrm{Jul} 25$ ];111(2):258-68. Available from: http://www.ncbi.nlm.nih.gov/pubmed/21272700.

24. Villanueva D, Marrugo J. De la dieta y sus metabolitos. RevMedicaSanitas. 2014;17(4):212-30.

25. Villanueva D. Influencia de los ácidos grasos poliinsaturados omega-3 y omega- 6 de la dieta y de sus metabolitos en la respuesta inmune de tipo alérgico. Rev Fac Med. 2015;63(2):301-13.

26. Torres-Borrego J, Moreno-Solís G, Molina-Terán AB. Diet for the prevention of asthma and allergies in early childhood: much ado about something? Allergol Immunopathol (Madr) [Internet]. 2012 [cited 2013 Mar 6];40(4):244-52. Available from: http://www.ncbi.nlm.nih.gov/ pubmed/22425606.

27. Harik-Khan RI. Serum Vitamin Levels and the risk of asthma in children. Am J Epidemiol [Internet]. 2004 Feb 15 [cited 2013 Mar 6];159(4):351-7. Available from: http://aje.oupjournals. org/cgi/doi/10.1093/aje/kwh053.

28. Pillai SG, Chiano MN, White NJ, Speer M, Barnes KC, Carlsen K, et al. A genome-wide search for linkage to asthma phenotypes in the genetics of asthma international network families: evidence for a major susceptibility locus on chromosome 2p. Eur J Hum Genet [Internet]. 2006 Mar [cited 2014 Jun 18];14(3):307-16. Available from: http://www.ncbi.nlm.nih.gov/ pubmed/16391567.

29. Taher Y, Van Esch M, Hofman G, Henricks J, van Oosterhout JM. 1,25-Dihydroxyvitamin D3 potentiates the beneficial effects of allergen immunotherapy in a mouse model of allergic asthma: role for IL-10 and TGF- J Immunol [Internet]. 2008;180(8):5211-21. Available from: http:// www.jimmunol.org/cgi/doi/10.4049/jimmunol.180.8.5211.

30. White $\mathrm{P}$, Cooke N. The multifunctional properties and characteristics of vitamin D-binding protein. Trends Endocrinol Metab [Internet]. 2000 Oct;11(8):320-7. Available from: http://www. ncbi.nlm.nih.gov/pubmed/10996527. 
31. Baeke F, Takiishi T, Korf H, Gysemans C, Mathieu C. Vitamin D : modulator of the immune system. Curr Opin Pharmacol [Internet]. Elsevier Ltd; 2010;10(4):482-96. Available from: http:// dx.doi.org/10.1016/j.coph.2010.04.001.

32. Kasiske BL. K/DOQI clinical practice guidelines for bone metabolism and disease in chronic kidney disease. [Internet]. Kasiske BL, editor. American Journal of kidney diseases : the official journal of the National Kidney Foundation. Minneapolis,MN55404: AJKD; 2003. S1-201 p. Available from: http://www.ncbi.nlm.nih.gov/pubmed/14520607.

33. Holick MF. Vitamin D deficiency. N Engl J Med [Internet]. 2007 Jul 19;357(3):266-81. Available from: http://www.ncbi.nlm.nih.gov/pubmed/17634462.

34. Pludowski P, Holick MF, Pilz S, Wagner CL, Hollis BW, Grant WB, et al. Vitamin D effects on musculoskeletal health, immunity, autoimmunity, cardiovascular disease, cancer, fertility, pregnancy, dementia and mortality-a review of recent evidence. Autoimmun Rev [Internet]. Elsevier B.V.; 2013 Aug [cited 2014 May 23];12(10):976-89. Available from: http://www.ncbi.nlm.nih.gov/ pubmed/23542507.

35. Quack M, Carlberg C. Ligand-triggered stabilization of vitamin D receptor/retinoid X receptor heterodimer conformations on DR4-type response elements. J Mol Biol [Internet]. 2000 Feb 25 [cited 2014 Jun 18];296(3):743-56. Available from: http://www.ncbi.nlm.nih.gov/pubmed/10677278.

36. Bikle DD, Teichert A, Arnold LA, Uchida Y, Elias PM, Oda Y. Differential regulation of epidermal function by VDR coactivators. J Steroid Biochem Mol Biol. 2010;121(1-2):308-13.

37. Carvallo L, Henriquez B, Olate J, van Wijnen AJ, Lian JB, Stein GS, et al. The 1alpha,25-dihydroxy Vitamin D3 receptor preferentially recruits the coactivator SRC-1 during up-regulation of the osteocalcin gene. J Steroid Biochem Mol Biol [Internet]. 2007 Mar [cited 2014 Jun 18];103(35):420-4. Available from: http://www.pubmedcentral.nih.gov/articlerender.fcgi?artid=3118563 \&tool=pmcentrez\&rendertype $=$ abstract.

38. Bikle DD, Teichert a, Arnold L a, Uchida Y, Elias PM, Oda Y. Differential regulation of epidermal function by VDR coactivators. J Steroid Biochem Mol Biol [Internet]. Elsevier Ltd; 2010 Jul [cited 2014 Jun 18];121(1-2):308-13. Available from: http://www.pubmedcentral.nih.gov/articlerender.fcgi?artid $=2906691$ \&tool $=$ pmcentrez\&rendertype $=$ abstract.

39. Rachez C, Freedman LP. Mechanisms of gene regulation by vitamin $D(3)$ receptor: a network of coactivator interactions. Gene [Internet]. 2000 Apr 4;246(1-2):9-21. Available from: http:// www.ncbi.nlm.nih.gov/pubmed/10767523.

40. Song Y, Hong J, Liu D, Lin Q, Lai G. 1,25-dihydroxyvitamin D3 inhibits nuclear factor kappa B activation by stabilizing inhibitor IKBa via mRNA stability and reduced phosphorylation in passively sensitized human airway smooth muscle cells. Scand J Immunol [Internet]. 2013 Feb [cited 2014 Jun 18];77(2):109-16. Available from: http://www.ncbi.nlm.nih.gov/pubmed/23126502.

41. Helming L, Böse J, Ehrchen J, Schiebe S, Frahm T, Geffers R, et al. 1alpha,25-Dihydroxyvitamin D3 is a potent suppressor of interferon gamma-mediated macrophage activation. Blood [Internet]. 2005 Dec 15 [cited 2014 Jun 3];106(13):4351-8. Available from: http://www.ncbi.nlm. nih.gov/pubmed/16118315.

42. Calton EK, Keane KN, Newsholme P, Soares MJ. The impact of Vitamin D levels on inflammatory status: A systematic review of immune cell studies. PLoS One. 2015;10(11):1-12.

43. Lagishetty $\mathrm{V}$, Liu NQ, Hewison M. Vitamin D metabolism and innate immunity. Mol Cell Endocrinol [Internet]. Elsevier Ireland Ltd; 2011 Dec 5 [cited 2014 May 25];347(1-2):97-105. Available from: http://www.pubmedcentral.nih.gov/articlerender.fcgi?artid=3200473\&tool=pmcentrez\&re ndertype $=$ abstract.

44. Riek $A E$, Oh J, Bernal-Mizrachi C. $1,25(\mathrm{OH}) 2$ vitamin $D$ suppresses macrophage migration and reverses atherogenic cholesterol metabolism in type 2 diabetic patients. J Steroid Biochem Mol Biol [Internet]. Elsevier Ltd; 2013 Jul [cited 2014 Jun 4];136:309-12. Available from: http:// www.ncbi.nlm.nih.gov/pubmed/23333932.

45. Penna G, Adorini L. 1 ,25-Dihydroxyvitamin D3 Inhibits Differentiation, Maturation, Activation, and Survival of Dendritic Cells Leading to Impaired Alloreactive T Cell Activation. J Immunol [Internet]. 2000 Mar 1 [cited 2014 Jun 18];164(5):2405-11. Available from: http://www.jimmunol. org/cgi/doi/10.4049/jimmunol.164.5.2405.

46. Adorini L, Penna G. Dendritic cell tolerogenicity: a key mechanism in immunomodulation by vitamin $D$ receptor agonists. Hum Immunol [Internet]. American Society for Histocompatibility and Immunogenetics; 2009 May [cited 2013 Mar 6];70(5):345-52. Available from: http://linkinghub.elsevier.com/retrieve/pii/S0198885909000214.

47. Froicu M, Weaver V, Wynn T a, McDowell MA, Welsh JE, Cantorna MT. A crucial role for the vitamin D receptor in experimental inflammatory bowel diseases. Mol Endocrinol [Internet]. 2003 Dec [cited 2014 Jun 18];17(12):2386-92. Available from: http://www.ncbi.nlm.nih.gov/pubmed/14500760.

48. Boonstra a, Barrat FJ, Crain C, Heath VL, Savelkoul HF, O'Garra a. 1alpha,25-Dihydroxyvitamin d3 has a direct effect on naive CD4(+) T cells to enhance the development of Th2 cells. J Immunol [Internet]. 2001 Nov 1;167(9):4974-80. Available from: http://www.ncbi.nlm.nih.gov/ pubmed/11673504.

49. Thoma-Uszynski S. Induction of direct antimicrobial activity through mammalian toll-Like receptors. Science (80- ) [Internet]. 2001;291(5508):1544-7. Available from: http://www.science- 
mag.org/cgi/doi/10.1126/science.291.5508.1544.

50. Liu PT, Stenger S, Li H, Wenzel L, Tan BH, Krutzik SR, et al. Toll-like receptor triggering of a vitamin D-mediated human antimicrobial response. Science [Internet]. 2006 Mar 24 [cited 2014 May 26];311(5768):1770-3. Available from: http://www.ncbi.nlm.nih.gov/pubmed/16497887.

51. Chun RF, Lauridsen AL, Suon L, Zella L a, Pike JW, Modlin RL, et al. Vitamin D-binding protein directs monocyte responses to 25-hydroxy- and 1,25-dihydroxyvitamin D. J Clin Endocrinol Metab [Internet]. $2010 \mathrm{Jul}$ [cited 2014 Jun 18];95(7):3368-76. Available from: http://www. pubmedcentral. nih.gov/articlerender.fcgi $?$ artid $=2928899 \&$ tool=pmcentrez\&rendertype $=$ abs tract.

52. Tissa R. Hata, Kotol P, Jackson M, Nguyen M, Paik A, Udall D, et al. Administration of oral vitamin $D$ induces cathelicidin production in atopic individuals. J Allergy Clin Immunol. $2008 ; 122(4): 828-9$.

53. Wang T-T, Nestel FP, Bourdeau V, Nagai Y, Wang Q, Liao J, et al. Cutting Edge: 1,25-Dihydroxyvitamin D3 is a direct inducer of antimicrobial peptide gene expression. ] Immunol [Internet]. 2004;173:2909-12. Available from: http://www.jimmunol.org/content/173/5/2909 nhttp://www.jimmunol.org/content/173/10/6490.4.full.pdf\nhttp://www.jimmunol.org/.

54. Karlsson J, Carlsson G, Larne O, Andersson M, Pütsep K. Vitamin D3 induces pro-LL-37 expression in myeloid precursors from patients with severe congenital neutropenia. J Leukoc Biol [Internet]. 2008 Nov [cited 2014 Jun 18];84(5):1279-86. Available from: http://www. ncbi.nlm.nih.gov/pubmed/18703682.

55. Veldman CM, Cantorna MT, Deluca HF. Expression of 1,25-Dihydroxyvitamin D 3 Receptor in the Immune System 1. 2000;374(2):334-8.

56. Mahon BD, Wittke A, Weaver V, Cantorna MT. The targets of vitamin D depend on the differentiation and activation status of CD4 positive T cells. J Cell Biochem [Internet]. 2003 Aug 1 [cited 2014 Jun 18];89(5):922-32. Available from: http://www.ncbi.nlm.nih.gov/pubmed/12874827.

57. Baeke F, Korf H, Overbergh L, van Etten E, Verstuyf A, Gysemans C, et al. Human T lymphocytes are direct targets of 1,25-dihydroxyvitamin D3 in the immune system. J Steroid Biochem Mol Biol [Internet]. Elsevier Ltd; 2010 Jul [cited 2014 Jun 18];121(1-2):221-7. Available from: http://www.ncbi.nlm.nih.gov/pubmed/20302932.

58. Rigby WF, Stacy T, Fanger MW. Inhibition of T lymphocyte mitogenesis by 1,25-dihydroxyvitamin D3 (calcitriol). J Clin Invest [Internet]. 1984 Oct;74(4):1451-5. Available from: http://www.pubmedcentral.nih.gov/articlerender.fcgi?artid=425314\&tool=pmcentrez\&rend ertype $=$ abstract.

59. Staeva-Vieira TP, Freedman LP. 1,25-Dihydroxyvitamin D3 Inhibits IFN- and IL-4 Levels During In Vitro Polarization of Primary Murine CD4+ T Cells. J Immunol [Internet]. 2002 Feb 1 [cited 2014 Jun 18];168(3):1181-9. Available from: http://www.jimmunol.org/cgi/ doi/10.4049/jimmunol.168.3.1181.

60. Jeffery LE, Burke F, Mura M, Zheng Y, Qureshi OS, Hewison M, et al. 1,25-Dihydroxyvitamin D3 and IL-2 combine to inhibit T cell production of inflammatory cytokines and promote development of regulatory T cells expressing CTLA-4 and FoxP3. J Immunol [Internet]. 2009 Nov 1 [cited 2014 May 27];183(9):5458-67. Available from: http://www.pubmedcentral. nih.gov/articlerender.fcgi?artid $=2810518$ \&tool=pmcentrez\&rendertype $=$ abstract.

61. Nanzer AM, Chambers ES, Ryanna K, Richards DF, Black C, Timms PM, et al. Enhanced production of IL-17A in patients with severe asthma is inhibited by 1a,25-dihydroxyvitamin D3 in a glucocorticoid-independent fashion. J Allergy Clin Immunol [Internet]. Elsevier Ltd; 2013 Aug [cited 2014 Jun 18];132(2):297-304.e3. Available from: http://www.ncbi.nlm. nih.gov/pubmed/23683514.

62. Joshi S, Pantalena L-C, Liu XK, Gaffen SL, Liu H, Rohowsky-Kochan C, et al. 1,25-dihydroxyvitamin $\mathrm{D}(3)$ ameliorates Th17 autoimmunity via transcriptional modulation of interleukin17A. Mol Cell Biol [Internet]. 2011 Sep [cited 2014 May 23];31(17):3653-69. Available from: http://www.pubmedcentral.nih.gov/articlerender.fcgi?artid=3165548\&tool=pmcentre z\&rendertype $=$ abstract.

63. Anolik $\mathrm{JH}$, Aringer $\mathrm{M}$. New treatments for SLE: cell-depleting and anti-cytokine therapies. Best Pract Res Clin Rheumatol [Internet]. 2005 Oct [cited 2014 Jun 18];19(5):859-78. Available from: http://www.ncbi.nlm.nih.gov/pubmed/16150407.

64. Heine G, Anton K, Henz BM, Worm M. 1alpha,25-dihydroxyvitamin D3 inhibits anti-CD40 plus IL-4-mediated IgE production in vitro. Eur J Immunol [Internet]. 2002 Dec;32(12):3395404. Available from: http://www.ncbi.nlm.nih.gov/pubmed/12432570.

65. Devereux G, Litonjua A a, Turner SW, Craig LC a, McNeill G, Martindale S, et al. Maternal vitamin $D$ intake during pregnancy and early childhood wheezing. Am J Clin Nutr [Internet]. 2007 Mar;85(3):853-9. Available from: http://www.ncbi.nlm.nih.gov/pubmed/17344509.

66. Sharief S, Jariwala S, Kumar J, Muntner P, Melamed ML. Vitamin D levels and food and environmental allergies in the United States: results from the National Health and Nutrition Examination Survey 2005-2006. J Allergy Clin Immunol [Internet]. Elsevier Ltd; 2011 May [cited 2014 May 30];127(5):1195-202. Available from: http://www.pubmedcentral.nih.gov/ articlerender.fcgi?artid $=3085636 \&$ tool $=$ pmcentrez\&rendertype $=$ abstract. 
67. Eyal Oren, Banerji A, Camargo CA. Vitamin D and atopic disorders in an obese population screened for vitamin D deficiency. J Allergy Clin Immunol [Internet]. 2008 Feb [cited 2014 May 27];121(2):531-3. Available from: http://www.ncbi.nlm.nih.gov/pubmed/18155280

68. Osborne NJ, Ukoumunne OC, Wake M, Allen KJ. Prevalence of eczema and food allergy is associated with latitude in Australia. J Allergy Clin Immunol. 2012;129(3):865-7.

69. Baïz N, Dargent-Molina P, Wark JD, Souberbielle J-C, Annesi-Maesano I. Cord serum 25-hydroxyvitamin $D$ and risk of early childhood transient wheezing and atopic dermatitis. ] Allergy Clin Immunol [Internet]. 2014 Jan [cited 2014 Jun 3];133(1):147-53. Available from: http://www.ncbi.nlm.nih.gov/pubmed/23810764.

70. Initiative G. Global strategy for asthma management and prevention. 2010;

71. Tolppanen A, Sayers A, Granell R, Fraser WD, Henderson J, Lawlor D a. Prospective Association of 25-Hydroxyvitamin D3 and D2 with Childhood Lung Function, Asthma, Wheezing, and Flexural Dermatitis. Epidemiology [Internet]. 2013;24(2):25-33. Available from: http://www.ncbi.nlm.nih.gov/pubmed/23377091.

72. Kolokotroni O, Papadopoulou A, Middleton N, Kouta C, Raftopoulos V, Nicolaidou P, et al. Vitamin $D$ levels and status amongst asthmatic and non-asthmatic adolescents in Cyprus: a comparative cross-sectional study. BMC Public Health [Internet] 2015;15(1):48. Available from: http://www.scopus.com/inward/record.url?eid=2-s2.084924232771\&partnerID=tZOtx3y1.

73. Erkkola M, Kaila M, Nwaru BI, Kronberg-Kippilä C, Ahonen S, Nevalainen J, et al. Maternal vitamin $\mathrm{D}$ intake during pregnancy is inversely associated with asthma and allergic rhinitis in 5-year-old children. Clin Exp Allergy [Internet]. 2009 Jun [cited 2014 May 28];39(6):875-82. Available from: http://www.ncbi.nlm.nih.gov/pubmed/19522996.

74. Freishtat RJ, Iqbal SF, Pillai DK, Klein CJ, Ryan LM, Benton AS, et al. High Prevalence of Vitamin D Deficiency among Inner-City African American Youth with Asthma in Washington, DC. J Pediatr [Internet]. Mosby, Inc.; 2010;156(6):948-52. Available from: http://dx.doi. org/10.1016/j.jpeds.2009.12.033.

75. Brehm JM, Celedón JC, Soto-Quiros ME, Avila L, Hunninghake GM, Forno E, et al. Serum vitamin D levels and markers of severity of childhood asthma in Costa Rica. Am J Respir Crit Care Med [Internet]. 2009 May 1 [cited 2013 Mar 6];179(9):765-71. Available from: http://www.pubmedcentral.nih.gov/articlerender.fcgi?artid=2675563\&tool=pmcentrez\&re ndertype $=$ abstract.

76. Elias PM, Hatano Y, Williams ML. Basis for the barrier abnormality in atopic dermatitis: outside-inside-outside pathogenic mechanisms. J Allergy Clin Immunol [Internet]. 2008 Jun [cited 2014 Jun 16];121(6):1337-43. Available from: http://www.pubmedcentral.nih. gov/articlerender.fcgi?artid =2706021\&tool=pmcentrez\&rendertype=abstract.

77. O'Regan GM, Sandilands A, McLean WHI, Irvine AD. Filaggrin in atopic dermatitis. J Allergy Clin Immunol [Internet]. 2008 Oct [cited 2014 May 23];122(4):689-93. Available from: http://www.ncbi.nlm.nih.gov/pubmed/18774165.

78. Gale CR, Robinson SM, Harvey NC, Javaid MK, Jiang B, Martyn CN, et al. Maternal vitamin D status during pregnancy and child outcomes. Eur ] Clin Nutr [Internet]. 2008 Jan [cited 2014 Jun 10];62(1):68-77. Available from: http://www.pubmedcentral.nih.gov/articlerender.fcgi?artid $=2629513 \&$ tool $=$ pmcentrez\&rendertype $=$ abstract.

79. Cheng HM, Kim S, Park G-H, Chang SE, Bang S, Won CH, et al. Low vitamin D levels are associated with atopic dermatitis, but not allergic rhinitis, asthma, or IgE sensitization, in the adult Korean population. J Allergy Clin Immunol [Internet]. Elsevier Ltd; $2014 \mathrm{Apr}$ [cited 2014 Jun 14];133(4):1048-55. Available from: http://www.ncbi.nlm.nih.gov/pubmed/24388009.

Universidad de Cartagena Fundada en 1827

REVISTA CIENCIAS BIOMÉDICAS
Es el órgano de información científica de la Facultad de Medicina de la Universidad de Cartagena. Colombia.

Publique su trabajo en esta revista enviando su manuscrito a: revistacienciasbiomedicas@unicartagena.edu.co www.revistacienciasbiomedicas.com

Revista ciencias Biomédicas es una publicación independiente, imparcial, abierta, revisada por pares, de elevada visibilidad internacional, con circulación online e impresa. Publica artículos en todas las modalidades universalmente aceptadas en inglés y en español, de temas referentes con todas las ciencias biomédicas, incluyendo ámbitos clínicos, epidemiológicos o de estudios básicos.

El sistema de gestión de manuscritos es rápido y justo. Revista Ciencias Biomédicas está incluida en varias bases de datos latinoamericanas e internacionales.

Antes de enviar su manuscrito, revise las recomendaciones para los autores, presentes en:

www. revistacienciasbiomedicas.com www.revistacienciasbiomedicas.com.co 